\title{
Call for Papers
}

\author{
Management and Organization Review \\ Special Issue on 'Building, Maintaining and Repairing Trust \\ across Cultures'
}

\author{
Guest Editors: \\ John Child, University of Birmingham, UK \\ Graham Dietz, Durham University, UK \\ Nicole Gillespie, Warwick University, UK \\ Peter Li, California State University, Stanislaus, US \\ Mark N. K. Saunders, Oxford Brookes University, UK \\ Denise Skinner, Coventry University, UK
}

Submission Deadline: December 1, 2008

Within the work setting, changes in work relationships and patterns (e.g., virtual teams, flatter hierarchies) and changes in societal expectations have accorded trust much greater significance in recent years. Yet, it remains unclear how trust is built, maintained and repaired across multiple interacting cultural groups of influence such as nations, industries, professions and organizations, as well as sub-cultures within organizations, and cultures based in ethnic and religious differences. Trust is understood to be central to human and group relations, and lies at the very foundation of society and civilization. Extant research and theory highlights how trust facilitates effective organizational performance through enhancing and enabling cooperation among disparate parties, open information sharing, mutual influence and acceptance, problem solving, commitment and job satisfaction, as well as a range of individual, group and organizational performance outcomes. The changing nature of organizations, their markets, employment patterns, and the social and political environments within which they operate necessitates an increased need for effective relationship building across different cultural groups or spheres. In particular, the increasing globalization of trade and transfers of capital and labour has meant that complex interdependencies within and between different organizations are developing, in the form of partnerships, networks and cross-boundary joint ventures. Trust is believed to be particularly important for success in complex environments characterized by high ambiguity and uncertainty, such as cross-cultural collaborations, yet the place and role of contextual 'culture' variables has, as yet, received limited attention, and the evidence presented thus far reports conflicting results.

With this special issue of $M O R$, we seek to further our understanding of trust and the ways in which it is built, maintained and repaired across cultures. We use the term culture in its broadest sense to encompass national, organizational, professional and industry 
cultures. Exploration of the issues relating to trust can, and do, take place in many settings and from a range of theoretical stances (e.g., economic, psychological, sociological). Rather than focusing on trust in a particular setting (e.g., public or private sector), we welcome contributions that address the theme of enabling trust across a broad range of cultural groups. The cross-cultural dimension of trust processes is critical to this special issue. It is intended that successful papers will add significantly to our understanding of the formation, maintenance and repair of trust within and across different cultures.

In keeping with the mission of $M O R$, papers addressing issues of trust in the Chinese context or that compare China to other cultural contexts would be very welcome. We invite submissions that address but are not limited to the following issues:

a) Trust at the interface of culturally distinct organizations. This might include businessto-business trust, as well as trust between business and other organizations, such as trade unions, government, the European Union, public sector organizations (e.g., public-private sector partnerships), religious bodies and professional networks.

b) Trust between organizational sub-cultures. Topics of interest here would include trust between organizational subcultures, hierarchies or professional subgroups.

c) The capacity of cultures to facilitate trust within and between organizations. How do cultures and organizations promote or impede trust?

d) How individuals accommodate and respond to the multiple cultural influences on their own attitudes and behaviours in relation to trust.

Questions about the special issue should be directed to Graham Dietz (graham.dietz@) durham.ac.uk), John Child (j.child@bham.edu.uk) or Anne Tsui (anne.tsui@asu.edu). Papers for the special issue should be submitted electronically to both Graham Dietz and the MOR office at iacmr.mor@asu.edu. 